Energy Research Journal 1 (2): 126-130, 2010

ISSN 1949-0151

(C) 2010 Science Publications

\title{
Arm Movement Recorder
}

\author{
Jakkrapun Chuanasa and Szathys Songschon \\ Department of Mechanical Engineering, \\ King Mongkut's University of Technology, Thonburi Bangkok, Thailand
}

\begin{abstract}
Problem statement: Human arm movement is widely studied nowadays. Some movements of arm are performed frequently in everyday life. Devices that can record the path of movement are important in studying human arm gesture. Approach: This project was performed the five degree-offreedom exoskeleton that was designed and constructed to observe position and orientation values of various movement in $x-y-z$ coordinates of human arm. All joints of the exoskeleton were attached with potentiometers for tracking a human arm trajectory. A dedicated computer with a software application, LabVIEW, obtained the data from arm movement and translated to the end effector coordinate. A calibration method for the exoskeleton was proposed for a particular path, a circle. A value correction for the chosen path was introduced in this calibration process and error of root mean square was used as performance index to measure radial error and geometric error of the exoskeleton. Results: Experiments with a human arm for executing repetitive tasks were performed to study. There were two sets of experiments. With correction factor applied to both experiments, the average Error of Root Mean Square (ERMS) reduced drastically. Conclusion: With the correction factor, this device could use to record particaular movement of human's arm with small error.
\end{abstract}

Key words: Exoskeleton, human arm, calibration

\section{INTRODUCTION}

In order to study motions of the human arm, many exoskeletons and orthoses are designed with sufficient principal Degrees Of Freedom (DOF) of the human arm. Most of the designs of the exoskeletons or orthoses are aimed to aid people with disabilities (Rahman et al., 2006; Rocon et al., 2007) and for rehabilitation (Carignan et al., 2005). A real-time monitoring system of measuring human upper limb movements in post-stroke rehabilitation (Zhou and $\mathrm{Hu}$, 2005) was proposed and represented by kinematic chain in which there are six joint variables to be considered: Three for the shoulder and the others for the elbow. Though the system is outstanding from any other existing 7 DOF limb models (Tolani et al., 2000; Rosen et al., 2005; Prokopenko et al., 2001), the elbow with three degrees of freedom is out of scope of this study. Recently, the design of 5 DOF exoskeleton has been proposed, the Maryland-Georgetown-Army (MGA) Exoskeleton (Carignan et al., 2005). The model of the MGA exoskeleton used the lowest number of DOFs possible to allow for full exercise therapy of the shoulder complex. The main goal of MGA exoskeleton is for exercise therapy and functional rehabilitation.

In this project, we adapted the design of MGA exoskeleton to record and study human arm movement. The forward kinematics (John, 2003) specifying the Cartesian position and orientation of the local frame attached to the human arm relative to the base frame was used to calculate the position and orientation of the end effector of the exoskeleton.

\section{MATERIALS AND METHODS}

The model of the exoskeleton with five DOF is shown in Fig. 1. There are sixteen parts and all parts made of resin. Figure 2 shows the exploded view of the model.

To describe the location of each link relative to its neighbors, the Denavit-Hartenverg (D-H) parameters are applied. By using D-H parameters, the position and orientation of the end effector of the exoskeleton can be calculated. The range of motion for each joints is given in Table 1. In order to measure the angles of each joints, potentiometers were needed to put to each location of the joints as shown in Fig. 3.

Corresponding Author: Jakkrapun Chuanasa, Department of Mechanical Engineering,

King Mongkut's University of Technology, Thonburi Bangkok, Thailand 


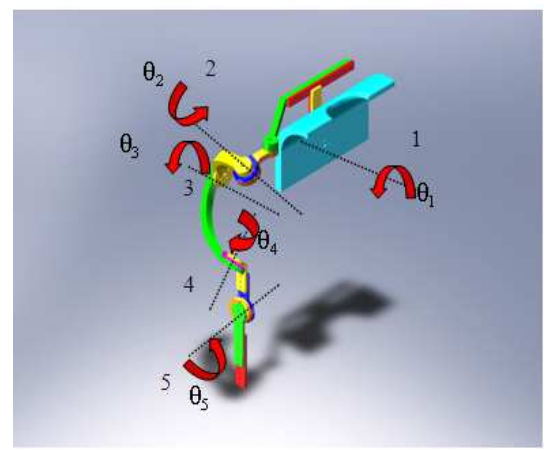

Fig. 1: Solidworks rendering of the exoskeleton

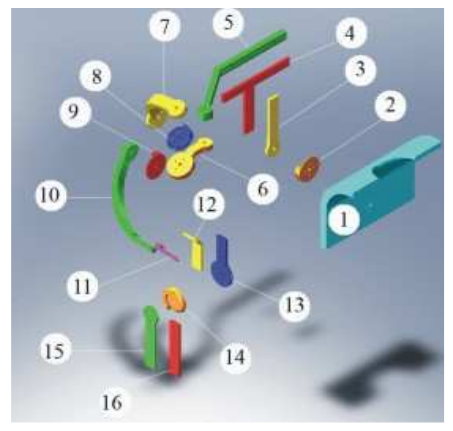

Fig. 2: The exploded view of the model

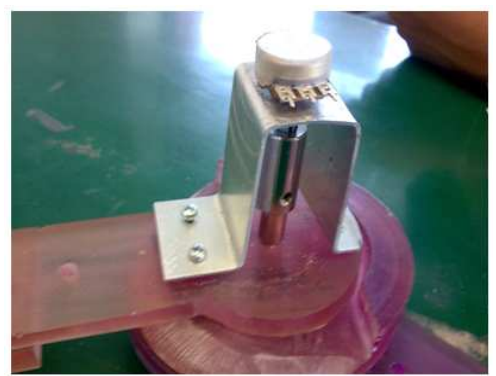

Fig. 3: Installing potentiometer to a joint

Table 1: Range of motion for each joints

\begin{tabular}{ll}
\hline Joint & Range (deg) \\
\hline Angle 1 & $+30 /-30$ \\
Angle 2 & $+180 /-45$ \\
Angle 3 & $+90 /-65$ \\
Angle 4 & $+210 /-30$ \\
Angle 5 & $+142 / 0$ \\
\hline Note: Angle 1-5 refer to each angle of the pivots as in Fig. 1
\end{tabular}

Note: Angle 1-5 refer to each angle of the pivots as in Fig. 1

All potentiometers are volume type which have the resistance of 5 kilo-ohms and they were calibrated with a 1,000 pulse/rev encoder. The reasons we used potentiometers instead of encoders were that the weight and cost of potentiometers are, respectively, lighter and cheaper compared to those of encoders.

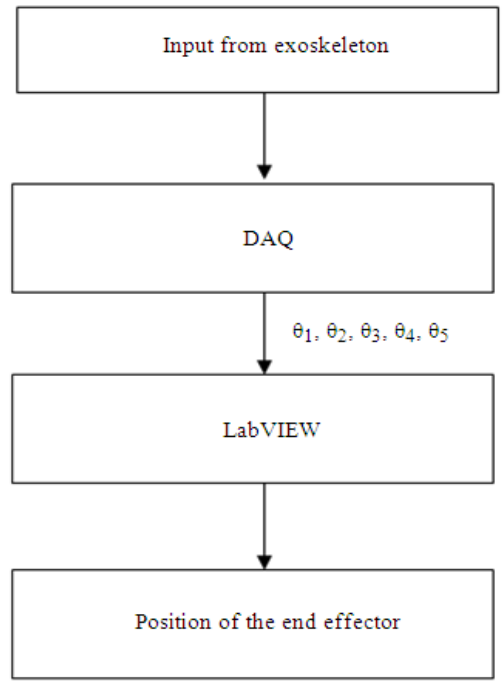

Fig. 4: Flow chart of the experiment

The experiment was designed as the following:

- Assigned a particular path for a subject to repeatedly move his/her arm that was attached with the exoskeleton (the path we used was a circle)

- Compared the positions of the end effector along the path of each time of experiment. After repeating the path for some particular times, the behavior of end effector could be analyzed.

LabVIEW was used in the experiment to measure the data. The steps of the experiment were as the following:

- A subject wore the exoskeleton

- The path that needed to be followed was a circle of 20 centimeters in radius

- The subject put the end effector at the starting point

- The record button in LabVIEW was turned on

- The subject moved the end effector along the provided path

- When the circle was fully drawn, the program in LabVIEW stopped and recorded the angles and computed for the positions

- The experiment was repeated for total 10 times

- The graphs of positions and angles of each potentiometers were plotted

- The errors were calculated

Figure 4 shows the flow chart of the experiment. $\theta_{1}, \theta_{2}, \theta_{3}, \theta_{4}$, and $\theta_{5}$ are, respectively, the angle 1 to angle 5 as mentioned in Table 1 with referring to Fig. 1. The exoskeleton was mounted on the subject as seen in Fig. 5. 


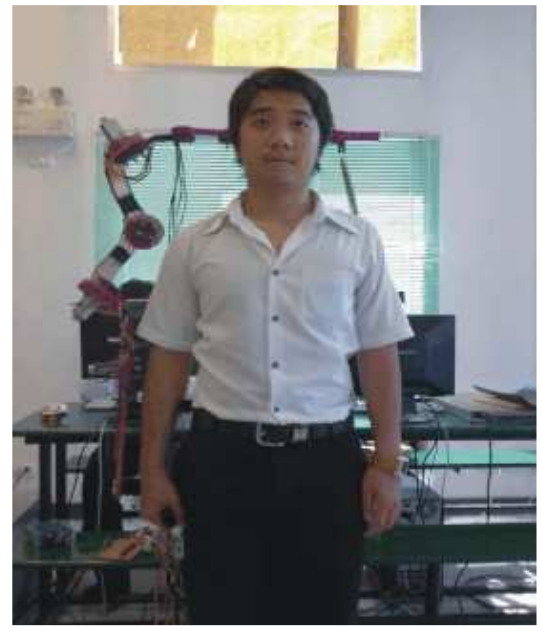

Fig. 5: A subject with the exoskeleton mounted

\section{RESULTS}

The experimental results of a subject after drawing a circle of 20 centimeters in radius for first time is shown in Fig. 6. The plot of angles for each potentiometers at any instant time of the first trial is shown in Fig. 7. The relationship between the radius and the angular displacement is shown in Fig. 8.

The errors for each angular displacement $\left(e_{n}\right)$ were obtained by subtracting the actual radius $\left(r_{a}\right)$ by the recorded radius $\left(r_{c}\right)$ :

$$
\mathrm{e}_{\mathrm{n}}=\mathrm{r}_{\mathrm{a}}-\mathrm{r}_{\mathrm{c}}
$$

Thus, the Error of Root Mean Square (ERMS) of each trial of the experiment can be written as:

$$
\text { erms }=\sqrt{\frac{e_{1}^{2}+e_{2}^{2}+e_{3}^{2}+\ldots+e_{n}^{2}}{n}}
$$

where, $\mathrm{n}$ is the number of the angular displacement.

The error for the first trial was $3.52 \mathrm{~cm}$. For 10 trials of the experiment, the average ERMS was 3.23 $\mathrm{cm}$ and the minimum ERMS was $3.03 \mathrm{~cm}$.

At each angular displacement, we used the correction factor which obtained from ERMS values to compensate the error movement of the exoskeleton. Fig. 9 shows the correction factor at each angular displacement. After we compensated the correction factor in the program, the experiment was repeated again and the plot of the first trial is shown in Fig. 10. The error for the first trial, this time, was $0.582 \mathrm{~cm}$. For 10 trials of the experiment, the average ERMS was $0.45 \mathrm{~cm}$ and the minimum ERMS was $0.32 \mathrm{~cm}$.

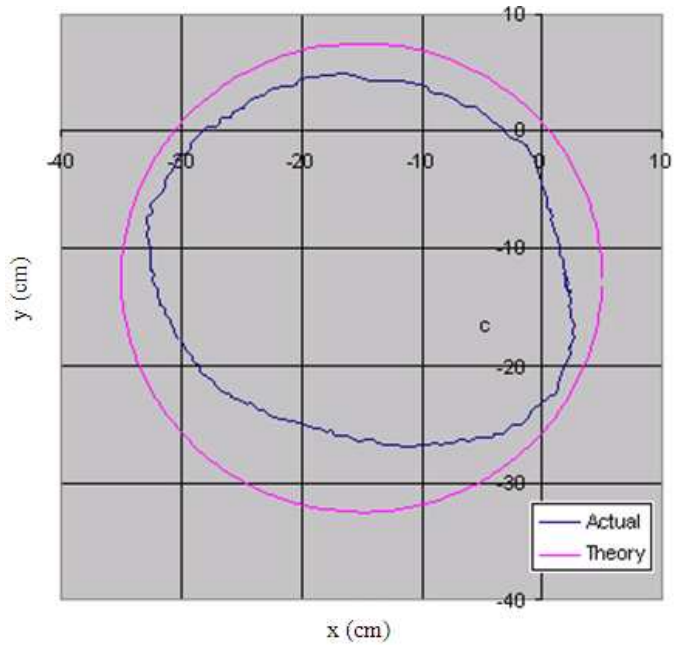

Fig. 6: Plot of movement in $\mathrm{x}$ and $\mathrm{y}$ coordinate of the first trial

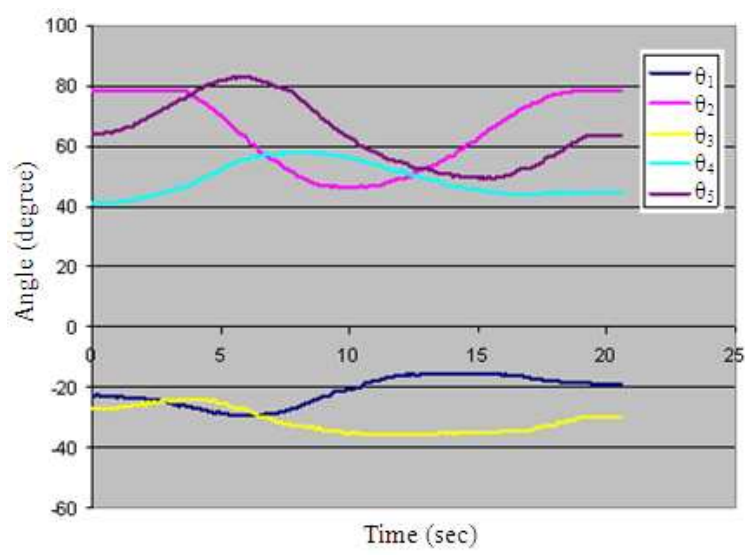

Fig. 7: The plot of angles of each potentiometer relative to time for the first trial

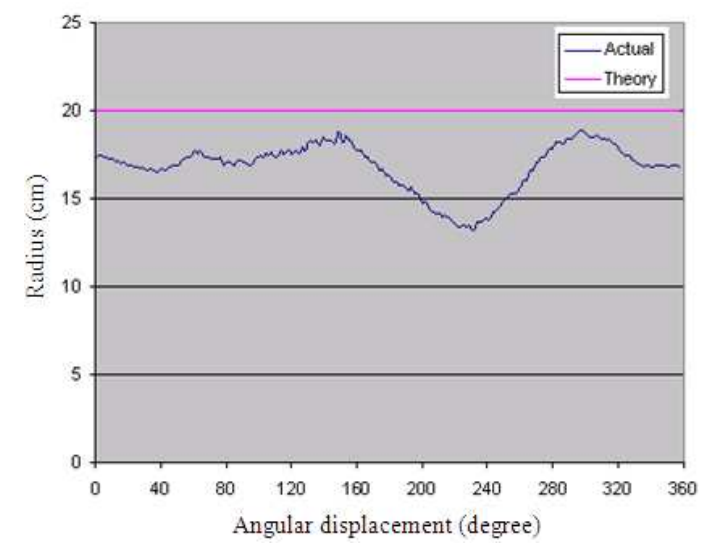

Fig. 8: The radius and angular displacement relationship 


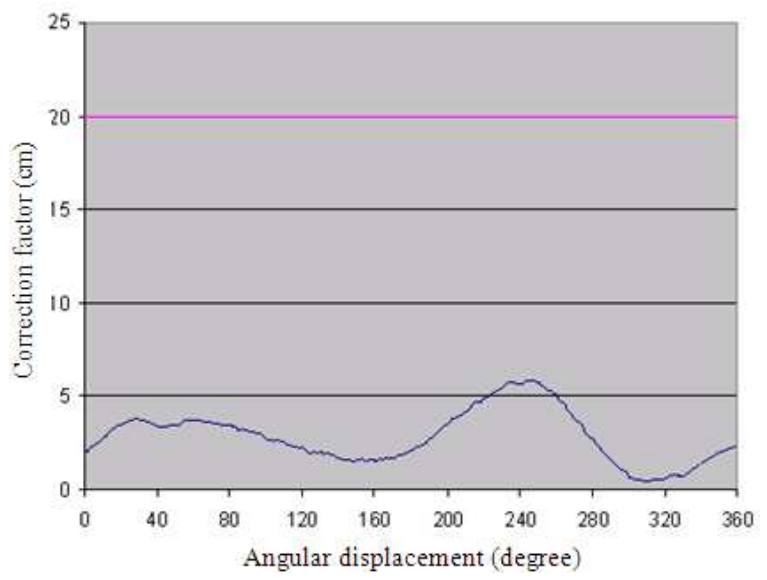

Fig. 9: The correction factor through the angular displacement

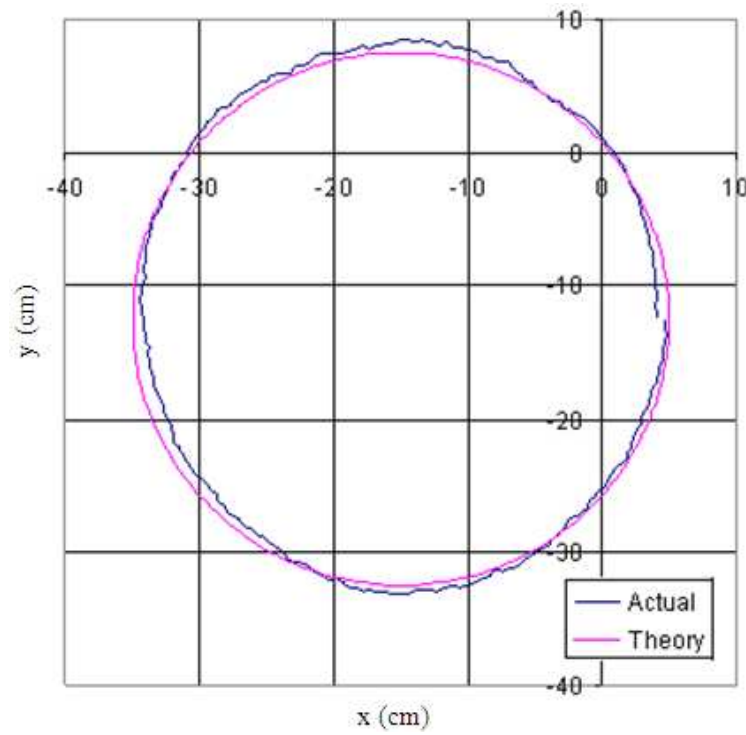

Fig. 10: First trial with correction factor

Another set of experiment was done with the same preparation but the radius of circle changed to $10 \mathrm{~cm}$. The average ERMS before the correction factor applied was $1.78 \mathrm{~cm}$. After the compensation, the average ERMS was $0.33 \mathrm{~cm}$.

\section{DISCUSSION}

The data shown in the plot (Fig. 6) is in $x-y-z$ coordinate which were calculated from the angular displacement of each potentiometers. Since the current for each potentiometers was from only one source, the voltage in each potentiometers read slightly different from what it used to be with the encoder calibration. Furthermore, when used for long time, potentiometers also read data inaccurately due to the heating effect.

Another problem was of the model itself. The model was made of resin which could be deformed under some amount of twisted torque. Some parts of the model were not exact in length and angle. All of these reason caused the experimental result errors.

\section{CONCLUSION}

For each trial of the experiment, the shape of the drawn circles look similar but smaller than the path of actual circle. To reduce the errors that occur from the potentiometers which have many problems as mentioned prior, encoders could be used instead. Nevertheless, the price of encoders is higher than potentiometers. For 10 trials of the experiment, the experiment of $20 \mathrm{~cm}$ radius circle had the ERMS decreased from 3.23-0.45 $\mathrm{cm}$ after applied the correction factor. Moreover, for the experiment of 10 $\mathrm{cm}$ radius circle, the average ERMS changed from $1.78-0.33 \mathrm{~cm}$. As the results shown, after the correction factor applied, the ERMS was drastically reduced.

The material of the model can be replaced with some other higher rigidity type of material rather than resin. However, the higher rigidity the material is, the higher the cost and the harder to reshape the model, especially the curve parts.

\section{ACKNOWLEDGMENT}

The researchers wish to thank Korakod Kleebyeesun, Kittipong Takoonwarasin, and Jakkrit Khobluang for their help in data collection and many other tasks done in this project.

\section{REFERENCES}

Carignan, C., M. Liszka and S. Roderick, 2005. Design of an exoskeleton with scapula motion for shoulder rehabilitation. Proceeding of the IEEE International Conference on Advanced Robotics, July 18-20, IEEE Xplore Press, Seattle, WA., pp: 524-531. DOI: 10.1109/ICAR.2005.1507459

John, J.C., 2003. Introduction to Robotics, Mechanics and Control. 3rd Edn., Prentice-Hall, USA., ISBN10: 0321223403, pp: 15-96.

Prokopenko, R.A., A.A. Frolov, E.V. Biryukova and A. Roby-Brami, 2001. Assessment of the accuracy of a human arm model with seven degrees of freedom. J. Biomech., 34: 177-85. DOI: 10.1016/S0021-9290(00)00179-2 
Rahman, T., W. Sample, S. Jayakumar, M.M. King, J.Y. Wee and R. Seliktar, 2006. Passive exoskeletons for assisting limb movement. J. Rehabil. Res. Dev., 43: 583-90. DOI: 10.1682/JRRD.2005.04.0070

Rocon, E., M. Manto, J. Pons, S. Camut and J.M. Belda, 2007. Mechanical suppression of essential tremor. Cerebellum, 6: 73-8. DOI: 10.1080/14734220601103037

Rosen, J., J.C. Perry, N. Manning, S. Burns and B. Hannaford, 2005. The human arm kinematics and dynamics during daily activities-toward a 7 DOF upper limb powered exoskeleton. Proceedings of the 2005 IEEE International Conference on Advanced Robotics, July 18-20, IEEE Xplore Press, Seattle, WA., pp: 532-539. DOI: 10.1109/ICAR.2005.1507460
Tolani, D., A. Goswami and N.I. Badler, 2000. Real-time inverse kinematics techniques for anthropomorphic limbs. Graphic. Models, 62: 353-388. DOI: 10.1006/gmod.2000.0528

Zhou, $\mathrm{H}$. and $\mathrm{H}$. $\mathrm{Hu}, 2005$. Inertial motion tracking of human arm movements in stroke rehabilitation. Proceeding of the IEEE International Conference on Mechatronics and Automation, July 29-Aug. 1, IEEE, Niagara Falls, Canada, pp: 1306-1311. http://cswww.essex.ac.uk/staff/hhu/Papers/IEEEICMA-057.pdf 